\title{
Self-Healing Solution To Heterogeneous Networks Using CoMP
}

\author{
Yu Jie ${ }^{1}$, Ahmad Alsharoa ${ }^{1}$, Ahmed Kamal ${ }^{1}$, and Mohammad Alnuem ${ }^{2}$ \\ ${ }^{1}$ Department of Electrical and Computer Engineering, Iowa State University (ISU), Ames, Iowa, USA, \\ Email: \{jysarah, alsharoa, kamal\}@iastate.edu \\ ${ }^{2}$ College of Computer and Information Science, King Saud University (KSU), Saudi Arabia, \\ Email: malnuem@ksu.edu.sa
}

\begin{abstract}
Self-healing mechanism is one of the three functionalities for self-organizing networks, and it has three major components to be studied by the academic society: fault detection, fault diagnosis and cell outage compensation. In this paper, we study the cell outage compensation function of the selfhealing mechanism. In a heterogeneous network environment with densely deployed Femto Base Stations (FBSs), we form a resource allocation problem for FBSs and Femto User Equipments (FUEs) operations using Coordinated Multi-Point (CoMP) transmission and reception with joint processing technique. Since the formulated problem is considered as NP hard problem, we propose a heuristic operation scheme to solve the problem. Simulation results show that our proposed operation scheme can improve FUE throughput by up to $30 \%$ compared to other solutions, and it can also prevent the system total rate loss from having the same speed of radio resource loss when failures happen.
\end{abstract}

Index Terms-Self-healing mechanism, heterogeneous network, coordinated multi-point.

\section{INTRODUCTION}

In modern cellular networks, subscribers peak data rates have been significantly improved with the introduction of mobile communication Long Term Evolution (LTE) standard and its further LTE-Advanced (LTE-A) evolution [1]. Meanwhile, mobile traffic is exponentially growing because of the broad use of smartphones, tablets together with the 'data hungry' applications. In order to meet the users requirements and increase spectrum efficiency and network capacity per area, mobile operators are deploying small cells overlaid with traditional macro cells to creat a Heterogeneous Network (HetNet) environment.

Small cells are low-powered radio access nodes that operate on operator-licensed band with a transmission range of 10 meters to 1-2 kilometers. There are different small cell categories such as micro cell, pico cell, femto cell. which are characterized by the transmission power, antenna gain and antenna height, etc. To successfully operate a large number of small cell BSs and reduce mobile service providers' network Operational Expenses (OPEX), enabling small cell BSs with self-organization capabilities is one promising solution.

This project was funded by the National Plan for Science, Technology and Innovation (MAARIFAH), King Abdulaziz City for Science and Technology, Kingdom of Saudi Arabia, Award Number 11-INF1920-02.
Self-organizing network functionalities are commonly divided into three main sub-functional groups [2]: selfconfiguration, self-optimization, and self-healing. In this paper, we study self-healing solutions in HetNets. When some nodes in the network become inoperative, self-healing mechanisms will automatically perform fault detection, fault compensation and recovery algorithms, aiming to reduce the impacts from the failure. Generally, self-healing solutions can be classified into three main categories: 1) fault detection, 2) fault diagnosis and 3) cell outage compensation. Cell outage compensation is performed by the system during the time period starting when the network failure happens to the point when the network back to normal operation, and it is supposed to compensate the network performance degradation by using other underutilized network resources. In most of the published research work, the commonly used cell outage compensation approaches are network element power management, antenna tilt, etc [3]-[7].

The main contributions of our paper can be summarized as follows: (i) Studying the cell outage compensation function of the self-healing solution. (ii) Proposing a cell outage compensation scheme use Coordinated Multi-Point (CoMP) transmission and reception [8], [9] wth joint processing technique (CoMP-JP) in networks with densely deployed small cell BSs, such as residential areas with densely deployed FBSs. The goal of the proposed cell outage compensation scheme is to mitigate failure impact without manual operation and simplify the auto-operation computation complexity.

The rest of the paper is organized as follows. Section II introduces the system model. The formulation of the resource allocation problem is given in Section III. Section IV explains the proposed solution to solve the resource allocation problem. The cell outage compensation solution is provided in Section V. The numerical results are discussed in Section VI. Finally, the paper is concluded in Section VII.

\section{System Model}

We consider a HetNet where Macro BS (MBS), Pico BSs (PBSs) and FBSs overlay with each other. FBSs are deployed within one dual strip apartment (apt) block [10]. The dual strip apartment block model is shown in Fig. 1, where each stripe has 2 rows of apartment building and each row has 10 apartment units. There is a 10 meters wide street separates the 


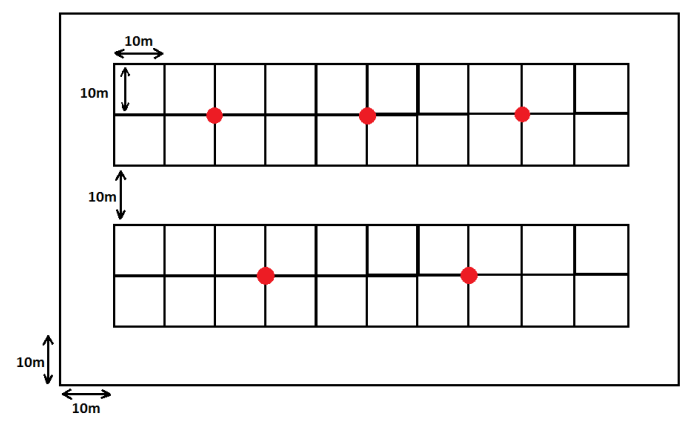

Fig. 1: Dual stripe apartment block model.

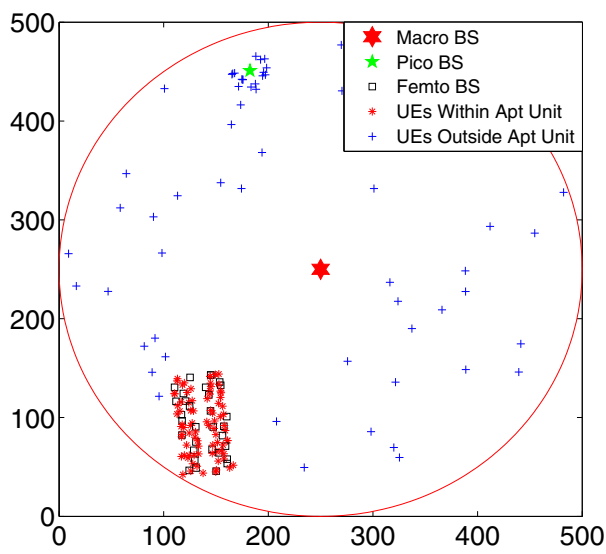

Fig. 2: A sample plot of system model.

two strip apartments. Fig. 2 plots a system model example with one MBS and one PBS. Users Equipment (UEs) (represented in blue +) are uniformly scattered over the field and a small amount of them are uniformly dropped within the coverage of PBS to simulate a hotspot. It is assumed that one dual strip apt block located within the field, and FBSs are randomly located within the apt units. There are also some UEs randomly deployed within each apt unit.

We define UEs located within apt units where a FBS is installed as FUEs and the FUE subscribes to the FBS within the same apt unit. Other UEs are called non-Femto UEs (nonFUEs). In this paper, we assume that FBSs operate in hybrid mode where they can be associated by FUEs that subscribe to other different FBSs, but not by UEs that do not subscribe to femto cellular service at all. We assume a perfect knowledge of the channel gains between the BSs and their served UEs are known to the BSs, and that each PBS, FBS, and UE has only one single radio antenna.

\section{Problem Formulation}

In this section, we formulate the CoMP-JP operation problem of all FBSs in the system into a resource allocation problem. The received signal at FUE $i$ from FBS $j$ is given by

$$
y_{i j}=h_{i j} x_{i j}
$$

where $h_{i j}$ and $x_{i j}$ are the channel gain between FBS $j$ and FUE $i$ and the signal transmitted from FBS $j$ towards FUE

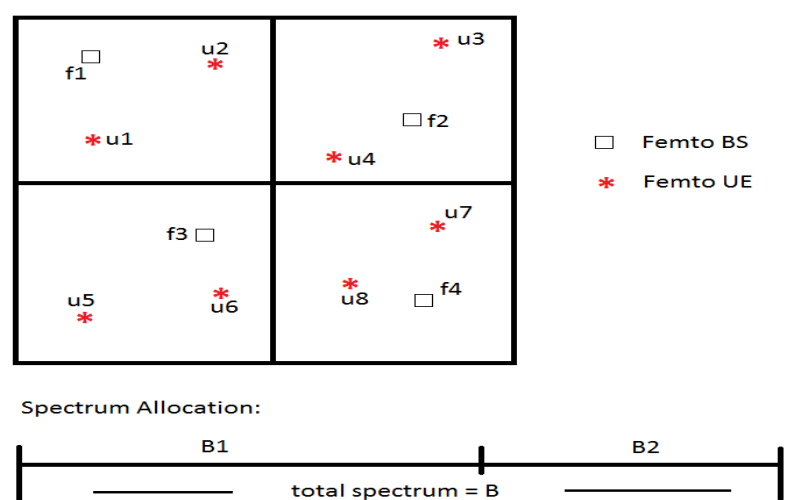

Fig. 3: A simple FBS CoMP-JP operation example.

$i$, respectively. By applying a linear precoding scheme on transmitted signals at FBSs, the transmitted signal becomes a linear transformation of the information symbols

$$
x_{i j}=t_{i j} s_{i}
$$

where $s_{i}$ and $t_{i j}$ are the information symbol for FUE $i$ and the precoding coefficient that FBS applied to information symbol $s_{i}$, respectively. It is assumed that $E\left\{s_{i} s_{i}^{H}\right\}=1$, where $(\cdot)^{H}$ denotes the conjugate transpose, The information symbols of different UEs are assumed to be orthogonal on each other.

In order to reduce intercell interference and improve system throughput, we allow FBSs in the apt to form non-overlapping clusters, and coordinate in providing service to groups of FUEs using CoMP-JP.

Example: A simple example is shown in Fig. 3 where the total spectrum is denoted by $B$. FBSs $\left\{f_{1}, f_{2}, f_{3}, f_{4}\right\}$ coorperate to provide service to FUEs $\left\{u_{1}, u_{2}, u_{3}, u_{4}, u_{5}, u_{6}, u_{7}, u_{8}\right\}$ using CoMP-JP. Since each FBS and FUE have one radio antenna, a FBS cluster $\mathcal{F}=\left\{f_{1}, f_{2}, f_{3}, f_{4}\right\}$ can only provide service to a set of FUEs $g$, such that $|\mathcal{F}| \geq|g|$, where $|\mathcal{F}|$ and $|g|$ are the cardinalities of sets $\mathcal{F}$ and $g$, respectively. In order to serve the 8 FUEs, we need to group the 8 FUEs into at least two sets and allocate to each set different spectrum. Therefore, we assume $g_{1}=\left\{u_{1}, u_{3}, u_{6}, u_{7}\right\}$ and $g_{2}=\left\{u_{2}, u_{4}, u_{5}, u_{8}\right\}$, and $B_{1}$ and $B_{2}$ are allocated to $g_{1}$ and $g_{2}$, respectively. After the scheduling, FBS cluster $\mathcal{F}$ can coordinate transmitting signals to $g_{1}$ on spectrum $B_{1}$, and $g_{2}$ on spectrum $B_{2}$ using CoMP-JP. $\left\{\mathcal{F}, g_{1}\right\},\left\{\mathcal{F}, g_{2}\right\}$ are two CoMP operation units.

Let the group of FUEs in one CoMP operation unit that contains FUE $i$ denoted by $g(i) . \mathcal{F}_{i}$ is the FBS cluster in the CoMP operation unit that provide service to $g(i)$. Using CoMP-JP, all FBSs within $\mathcal{F}_{i}$ transmit a linear combination of information symbols for all FUEs in $g(i)$. The transmitted signal of each FBS $j$ within $\mathcal{F}_{i}$ is given by

$$
x_{j}=\sum_{k \in g(i)} t_{k j} s_{k}
$$

The received signal at FUE $i$ is therefore given by

$$
\begin{aligned}
y_{i} & =\sum_{j \in \mathcal{F}_{i}} h_{i j} x_{j}+Z_{i} \\
& =\sum_{j \in \mathcal{F}_{i}} \sum_{k \in g(i)} h_{i j} t_{k j} s_{k}+Z_{i}
\end{aligned}
$$


where $Z_{i}$ combines the intercluster interference and noise for FUE $i$.

By applying zero forcing linear precoding technique on the transmitted signals, the interference to FUE $i$ caused by information symbols of other UEs within $g(i)$ can be cancelled [11]. The following should be satisfied when designing the precoding coefficients on transmitted information symbols

$$
\sum_{j \in \mathcal{F}_{i}} \sum_{k \in g(i), k \neq i} h_{i j} t_{k j} s_{k}=0
$$

since $s_{k}$ is orthogonal with $s_{l}, \forall k, l \in g(i), k \neq l$, we have

$$
\sum_{j \in \mathcal{F}_{i}} h_{i j} t_{k j}=0 \quad \forall k \in g(i), k \neq i
$$

By combining (5) and (7), we have

$$
y_{i}=\sum_{j \in \mathcal{F}_{i}} h_{i j} t_{i j} s_{i}+Z_{i}
$$

The precoding coefficients are designed such that (7) and (8) can be satisfied for all FUEs within $g(i)$.

LTE and LTE-A spectrum are assigned in unit of resource blocks. Assume $B_{0}$ is the bandwidth of one resource block. $R B_{g(i)}$ denotes the number of resource blocks assigned to FUE set $g(i)$ by FBS cluster $\mathcal{F}_{i}$. According to ShannonHartley theorem, the data rate of FUE $i$ is given by

$$
\text { Rate }_{i}=R B_{g(i)} B_{0} \log _{2}\left(1+\frac{\left|\sum_{j \in \mathcal{F}_{i}} h_{i j} t_{i j}\right|^{2}}{I_{i} R B_{g(i)} B_{0}}\right)
$$

where $I_{i}$ denotes as the interference plus noise spectrum density for FUE $i$. The operation of each FBS $j$ in the system is subject to power and spectrum constraints. Let $\mathcal{F}(j)$ be a FBS cluster that contains FBS $j$, and the set of FUEs served by $\mathcal{F}(j)$ are scheduled into different FUE groups $\mathcal{G}_{j}=\left\{g_{1}, g_{2}, \cdots, g_{\left|\mathcal{G}_{j}\right|}\right\}$ where $\left|\mathcal{G}_{j}\right|$ is the cardinality of set $\mathcal{G}_{j}$. Each FUE group $g_{k} \in \mathcal{G}_{j}$ together with $\mathcal{F}(j)$ forms one CoMP operation unit. Therefore, $\mathcal{U}_{j}=g_{1} \cup g_{2} \cup \cdots \cup g_{\left|\mathcal{G}_{j}\right|}$ is the set of total FUEs that are served by $\mathcal{F}(j)$. In the example of Fig. 3, for FBS $f_{1}, \mathcal{F}(1)=\left\{f_{1}, f_{2}, f_{3}, f_{4}\right\}$, and $\mathcal{G}_{1}=\left\{g_{1}, g_{2}\right\}$, and its total served FUEs set is $\mathcal{U}_{1}=g_{1} \cup g_{2}=$ $\{u 1, u 2, u 3, u 4, u 5, u 6, u 7, u 8\}$.

Let $P_{j}$ be the maximum transmit power of $\operatorname{FBS} j$, then

$$
\sum_{k \in \mathcal{U}_{j}}\left|t_{k j}\right|^{2} \leq P_{j}
$$

Let $R B_{g_{k}}$ be the number of resource blocks assigned to the FUE group $g_{k} \in \mathcal{G}_{j}$ by the FBS cluster $\mathcal{F}(j)$, then we have

$$
\sum_{g_{k} \in \mathcal{G}_{j}} R B_{g_{k}} \leq R B_{m}^{\max } \quad \forall m \in \mathcal{F}(j)
$$

where $R B_{m}^{\max }$ is the maximum available number of resource blocks to FBS $m$.

Finally, our resource allocation problem that maximize FUEs throughput can be formulated as follows

$$
\underset{\substack{t_{i j}, R B_{g_{k}}, \mathcal{G}_{j}, g(i), \mathcal{F}(j), \mathcal{F}_{i}, \forall i \in \mathcal{U}, \forall j \in \mathcal{M}}}{\operatorname{maximize}} \quad \sum_{i \in \mathcal{U}} \text { Rate }_{i}
$$

subject to:

$$
\begin{aligned}
& \sum_{i \in \mathcal{U}_{j}}\left|t_{i j}\right|^{2} \leq P_{j}, \quad \forall j \in \mathcal{M} \\
& \sum_{g_{k} \in \mathcal{G}_{j}} R B_{g_{k}} \leq \min _{m \in \mathcal{F}(j)} R B_{m}^{\text {max }}, \quad \forall j \in \mathcal{M} \\
& \sum_{m \in \mathcal{F}_{i}} h_{i m} t_{k m}=0, \quad \forall k \in g(i), k \neq i, \forall i \in \mathcal{U}
\end{aligned}
$$

where $\mathcal{M}$ is the set of all FBSs in the system, $\mathcal{U}$ is the set of all FUEs in the system. Constraints (13) and (14) are the power constraint and spectrum constraint for each FBS, respectively. While, constraint (15) is the zero-forcing precoding requirement for each FUE. $\left(\mathcal{F}(j), \mathcal{G}_{j}\right)$ and $\left(\mathcal{F}_{i}, g(i)\right)$ are (FBS cluster, FUE group) defined from FBS and FUE point of view, respectively. This problem is considered NP-hard mixed integer mathematical programing problem, and it is difficult to solve, especially in practical situations as the size of FBSs and FUEs is large. In the sequel, we propose an efficient heuristic scheme that decompose the problem into sub-problems and solve each sub-problem separately.

\section{Proposed CoMP-JP Operation Scheme}

In this section, we propose a heuristic way (divide and conquer) to solve the formulated problem given in (12)-(15), where we need to find FBSs and FUEs CoMP operation units, precoding coefficients for FBSs as well as resource blocks allocation to optimize the objective function. The FBSs and FUEs CoMP operation units formation involves FBS clustering and FUE scheduling. Firstly, we perform FBS clustering and FUE scheduling given the FBSs and FUEs topology and transmission channel conditions. Afterwards, the CoMP operation units can be formed. We then design the precoding coefficients for all FBSs within each CoMP operation unit such that the throughput of the CoMP operation unit is maximized. Finally, spectrum allocation is performed based on the performance of each CoMP operation unit.

\section{A. FBS clustering and FUE scheduling}

FBS with low transmission power has a transmission range of 20-30 meters. Based on the FBSs-FUEs deployment topology and FBSs' low transmission power feature, we can perform FBS clustering as shown in Fig. 1. We first locate the red spots in the center of each dense femto service traffic area and their locations will accommodate the traffic dynamics. The locations of the red spots should be properly spaced and be at least three to four apt units away from each other. With the red spots locations, the FBS clustering and FUE scheduling process can be implemented in following steps

Step (1): FBS clustering. Find the $N_{c}$ UEs $\left\{e_{1}, e_{2}, \cdots, e_{N_{c}}\right\}$ that are closest to the $N_{c}$ red spots locations, respectively. For each UE $e_{i}, \forall i \in\left\{1,2, \cdots, N_{c}\right\}$, form a FBS cluster $\mathcal{C}_{i}$ of size $S$ that have the strongest signal power toward UE $e_{i} . S$ is an integer number given by users. Let $\mathcal{C}=\left\{\mathcal{C}_{1}, \mathcal{C}_{2}, \cdots, \mathcal{C}_{N_{c}}\right\}$ be the set of clusters in the system.

Step (2): FBS clustering refinement. Step (1) may generate clusters that have the same FBS existing in two or more 
clusters, or have some FBSs not assigned to any cluster. If there exists FBS $j$ that satisfies either of these two conditions, assign it to cluster $\mathcal{C}_{i}$, where it has the strongest average perUE channel gain towards UEs located in the same apt units of FBSs in $\mathcal{C}_{i}$.

$$
\mathcal{C}_{i}=\arg \max _{\mathcal{C}_{i} \in \mathcal{C}} \frac{\sum_{k \in \mathcal{U}\left(\mathcal{C}_{i}\right)}\left|h_{k j}\right|^{2}}{\left|\mathcal{U}\left(\mathcal{C}_{i}\right)\right|}
$$

where $\mathcal{U}\left(\mathcal{C}_{i}\right)$ is the set of UEs located within apt units that deploy FBSs of $\mathcal{C}_{i} . h_{k j}$ is the channel gain of FBS $j$ towards UE $k$.

Step (3): grouping FUEs. Define $\mathcal{U}_{i}$ as the set of FUEs assigned to be served by FBS cluster $\mathcal{C}_{i}$, and an anchor FBS [12] of a FUE $k$ as the FBS that has the strongest signal power towards the UE. Then $\mathcal{U}_{i}$ is the set of FUEs that their anchor FBSs are within the FBS cluster $\mathcal{C}_{i}$.

Step (4): FUE scheduling. For each $\mathcal{C}_{i} \in \mathcal{C}$, divide its UE set $\mathcal{U}_{i}$ into subgroups $\mathcal{G}_{i}=\left\{g_{1}, g_{2}, \cdots, g_{\left|\mathcal{G}_{i}\right|}\right\}$, with each subgroup having a UE size which is the same as the FBS size of the cluster $\mathcal{C}_{i}$ :

$$
\left|\mathcal{G}_{i}\right|=\left\lceil\frac{\left|\mathcal{U}_{i}\right|}{\left|\mathcal{C}_{i}\right|}\right\rceil
$$

the dividing process of $\mathcal{U}_{i}$ is random, and we just need to make sure the maximum subgroup size is $\left|\mathcal{C}_{i}\right|$. The size of one subgroup can be less than $\left|\mathcal{C}_{i}\right|$. Now FUE set $\mathcal{U}_{i}$ is scheduled into $\left|\mathcal{G}_{i}\right|$ subgroups, and each subgroup $g_{k} \in \mathcal{G}_{i}$ together with the FBS cluster $\mathcal{C}_{i}$ form one CoMP operation unit.

\section{B. Power Allocation and Precoding Coefficients Calculation}

After FBS clustering and FUE scheduling, we reformulate problem (12)-(15) as follows

$$
\begin{array}{ll}
\underset{\substack{t_{k m}, R B_{g_{j}}, \forall k \in g_{j}, \forall g_{j} \in \mathcal{G}_{i}, \forall m \in \mathcal{C}_{i}, \forall \mathcal{C}_{i} \in \mathcal{C}}}{\operatorname{maximize}} \sum_{\mathcal{C}_{i} \in \mathcal{C}} \sum_{g_{j} \in \mathcal{G}_{i}} R B_{g_{j}} B_{0} . \\
& \sum_{k \in g_{j}} \log _{2}\left(1+\frac{\left|\sum_{m \in \mathcal{C}_{i}} h_{k m} t_{k m}\right|^{2}}{I_{k} R B_{g_{j}} B_{0}}\right)
\end{array}
$$

subject to:

$$
\begin{gathered}
\sum_{g_{j} \in \mathcal{G}_{i}} \sum_{k \in g_{j}}\left|t_{k m}\right|^{2} \leq P_{m}, \quad \forall m \in \mathcal{C}_{i}, \forall \mathcal{C}_{i} \in \mathcal{C} \\
\sum_{g_{j} \in \mathcal{G}_{i}} R B_{g_{j}} \leq \min _{m \in \mathcal{C}_{i}} R B_{m}^{\text {max }}, \quad \forall \mathcal{C}_{i} \in \mathcal{C} \\
\sum_{m \in \mathcal{C}_{i}} h_{k m} t_{l m}=0, \forall l \in g_{j}, l \neq k, \forall k \in g_{j}, \forall g_{j} \in \mathcal{G}_{i}, \forall \mathcal{C}_{i} \in \mathcal{C}
\end{gathered}
$$

The problem is still nonconvex, mixed integer mathematical programming problem. We can further decompose the problem. We first consider solving the above problem by setting $R B_{g_{j}}=1, \forall g_{j} \in \mathcal{G}_{i}, \forall i \in\left\{1,2, \cdots, N_{c}\right\}$, which is to maximize the sum rate of all CoMP operation units in the system on one resource block. We then do resource allocation on each CoMP operation unit later on.

After setting the $R B_{g_{j}}=1, \forall g_{j} \in \mathcal{G}_{i}, \forall i \in\left\{1,2, \cdots, N_{c}\right\}$, the resulting problem can be divided into $\sum_{i \in\left\{1,2, \cdots, N_{c}\right\}}\left|\mathcal{G}_{i}\right|$ subproblems, and one such subproblem of maximizing the throughput of one CoMP operation unit (FBS cluster $\mathcal{C}_{i}$, FUE group $g_{j} \in \mathcal{G}_{i}$ ) on one resource block is as follows

$$
\underset{\substack{t_{k m}, \forall k \in g_{j} \\ \forall m \in \mathcal{C}_{i}}}{\operatorname{maximize}} \sum_{k \in g_{j}} B_{0} \log _{2}\left(1+\frac{\left|\sum_{m \in \mathcal{C}_{i}} h_{k m} t_{k m}\right|^{2}}{I_{k} B_{0}}\right)
$$

subject to:

$$
\begin{aligned}
& \sum_{k \in g_{j}}\left|t_{k m}\right|^{2} \leq \frac{P_{m}}{R B_{m}^{\text {max }}}, \quad \forall m \in \mathcal{C}_{i} \\
& \sum_{m \in \mathcal{C}_{i}} h_{k m} t_{l m}=0, \quad \forall l \in g_{j}, l \neq k, \forall k \in g_{j}
\end{aligned}
$$

We assume that the FBS spreads its power on its available resource blocks evenly. Therefore, equation (23) has a power constraint of $\frac{P_{m}}{R B_{m}^{\max }}$. In our model, we assume $R B_{m}^{\max }, \forall m \in$ $\mathcal{C}_{i}, \forall \mathcal{C}_{i} \in \mathcal{C}$ are the same, and are defined as $R B^{\max }$. Problem (22)-(24) can be converted to a convex optimization problem according to reference [11]. Given perfect knowledge of the channel conditions between FBSs and FUEs, we can solve problem (22)-(24) using CVX [13] or other convex problem solvers.

\section{Resource Allocation}

We consider proportional fairness on the resource allocation problem. For a FBS cluster $\mathcal{C}_{i}$, the resource blocks scheduled to each UE group $g_{j} \in \mathcal{G}_{i}$ are proportional to spectrum efficiency sum of all UEs in each group $g_{j}$. Let $S E_{g_{j}}$ be spectrum efficiency sum of all UEs within group $g_{j}$. Then

$$
S E_{g_{j}}=\sum_{k \in g_{j}} \log \left(1+S I N R_{k}\right) \quad \forall g_{j} \in \mathcal{G}_{i}
$$

where $S I N R_{k}$ is the signal to interference plus noise ratio of FUE $k$. The number of resource blocks assigned to each group of FUEs is

$$
R B_{g_{j}}=\left\lfloor R B^{\max } \frac{S E_{g_{j}}}{\sum_{g_{k} \in \mathcal{G}_{i}} S E_{g_{k}}}\right\rfloor \quad \forall g_{j} \in \mathcal{G}_{i}
$$

The remaining resource blocks $R B^{\max }-\sum_{g_{j} \in \mathcal{G}_{i}} R B_{g_{j}}$ will be added to each subgroup in a round-robin way.

\section{Cell Outage Compensation Solution}

In our CoMP operation unit formation, clusters of FBSs are formed independently from each other, and the FUEs sets of any two CoMP operation units are nonoverlapping. If one FBS suffers from any failure, it will only affect the operation of the CoMP operation units it works in, meanwhile reduce the intercluster interference to FUEs within other CoMP operation units.

To perform quick failure compensation, the affected CoMP operation units will maintain their FBS clustering groups 
TABLE I: FUE average rate loss with different FBS failure probability

\begin{tabular}{|c|c|c|c|c|c|c|c|c|c|c|c|c|c|c|c|}
\hline Failure Probability & 0 & 0.01 & 0.02 & 0.03 & 0.04 & 0.05 & 0.06 & 0.07 & 0.08 & 0.09 & 0.1 & 0.2 & 0.3 & 0.4 & 0.5 \\
\hline Rate Loss & 0 & 0.019 & 0.010 & 0.025 & 0.043 & 0.039 & 0.048 & 0.06 & 0.079 & 0.065 & 0.075 & 0.123 & 0.187 & 0.244 & 0.334 \\
\hline
\end{tabular}

unchanged, but re-perform FUEs scheduling, precoding coefficients calculation as well as resource allocation as introduced in previous sections. For example, in Fig. 3, we have two CoMP operation units: $\left\{\mathcal{C}_{1}, g_{1}\right\},\left\{\mathcal{C}_{1}, g_{2}\right\}$, where $\mathcal{C}_{1}$ is the FBS cluster $\left\{f_{1}, f_{2}, f_{3}, f_{4}\right\}, g_{1}$ and $g_{2}$ are the FUEs groups $\left\{u_{1}, u_{3}, u_{6}, u_{7}\right\}$ and $\left\{u_{2}, u_{4}, u_{5}, u_{8}\right\}$, respectively. When FBS $f_{1}$ fails, we maintain the FBSs $\mathcal{C}_{1}=\left\{f_{2}, f_{3}, f_{4}\right\}$ as the new cluster, and re-group the FUEs using the proposed scheduling scheme in Section IV. In this example, we have three groups: $g_{1}=\left\{u_{1}, u_{3}, u_{7}\right\}, g_{2}=\left\{u_{2}, u_{6}, u_{8}\right\}, g_{3}=\left\{u_{4}, u_{5}\right\}$. Therefore, three different CoMP operation units are formed: $\left\{\mathcal{C}_{1}, g_{1}\right\},\left\{\mathcal{C}_{1}, g_{2}\right\},\left\{\mathcal{C}_{1}, g_{3}\right\}$, and each of which has to perform precoding coefficients calculation as well as resource allocation before they can operate successfully.

The two different cases considered in real implementation of failure compensation are given as follows:

Case 1: At least one FBS in the FBS cluster suffering from failure is still functional. The compensation operation is: keep the FBS cluster unchanged, and re-perform UE scheduling, precoding coefficients calculation and resource allocation.

Case 2: All FBSs within the FBS cluster fail. Then the FUEs served by the FBS cluster be handed over to the MBS or PBS with the strongest signal strength.

\section{NUMERICAL RESULTS}

In this section, we study the performance of our proposed CoMP-JP operation scheme. In MATLAB platform, we set up one MBS, one PBS and one dual strip apt block. Each apt unit has 0.8 probability of installing one FBS. We deploy two UEs in every apt units (80 UEs in total located within the apt block). There are another 60 UEs deployed outside the apt block. UEs deployed outside the apt block or deployed in apt units that have no FBS installed can only associate with MBS or PBS.

The simulation has a carrier center frequency of $2 \mathrm{GHz}$, and $10 \mathrm{MHz}$ total spectrum bandwidth. The transmission powers for FBS, PBS and MBS are $20 \mathrm{dBm}, 37 \mathrm{dBm}$, and $46 \mathrm{dBm}$, respectively, and their corresponding antenna gains are $5 \mathrm{dBi}$, $5 \mathrm{dBi}$, and $15 \mathrm{dBi}$, respectively. The $\mathrm{UE}$ antenna gain is 0 $\mathrm{dBi}$. The noise power spectrum density is $-174 \mathrm{dBm} / \mathrm{Hz}$. The propagation loss model, shadowing fading model and the penetration loss model between BSs and UEs are taken from reference [10] (refer to [10] for more details).

For comparison purpose, we apply two other solutions to the same simulation setup:

Comparison solution 1 (CSolu1): in normal operation mode, a FUE will associate with the FBS located within the same apt unit as the FUE. Other non-FUEs will associate with the MBS or PBS with the highest signal strength. When a FBS failure happens, the FUEs suffering from failure will associate with the BS (FBSs that are still functional, PBS or MBS) that provide them with the strongest signal power strength.

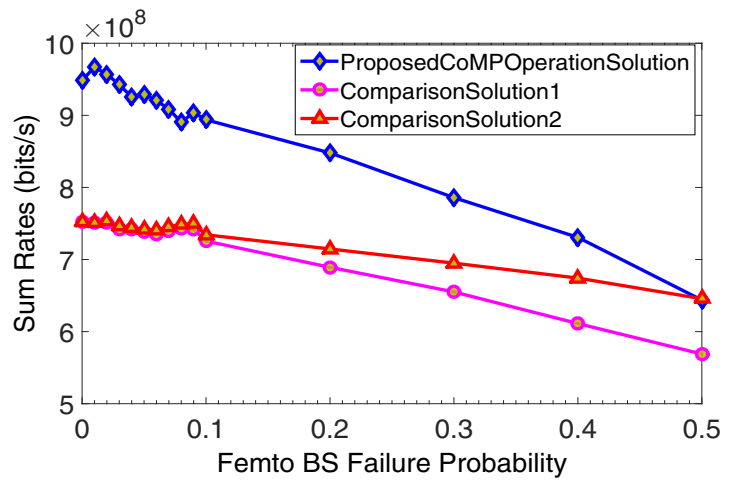

Fig. 4: Sum rates of FUEs within the residential apt units under different FBS failure probabilities.

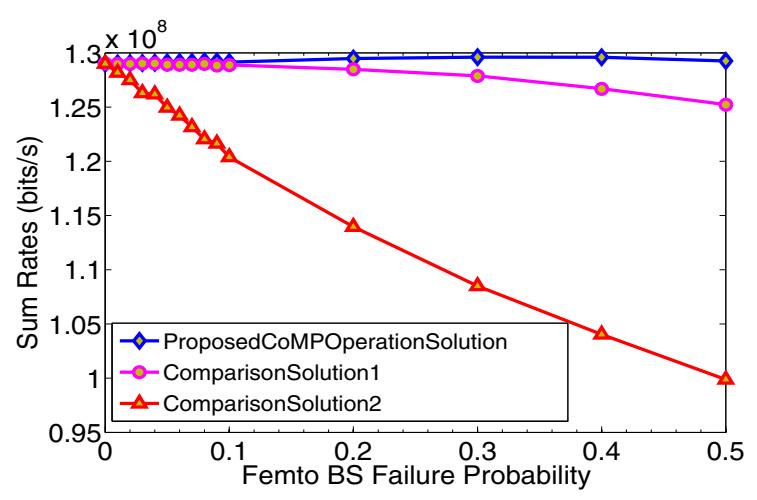

Fig. 5: Sum rates of non-FUEs outside the residential apt units under different FBS failure probabilities.

Comparison solution 2 (CSolu2): in normal operation mode, the UEs behave the same as in CSolu1. However, when a FBS failure happens, the FUEs suffering from failure will only associate with MBS or PBS with highest signal strength.

We assume the system environment is quasi-static (the devices locations and the channel gains remain static during the process of calculation). For a given simulated system environment, we apply our proposed CoMP-JP operation scheme and the CSolu1 and CSolu2 under different FBS failure probabilities, and calculate the achievable rate of each UE within the system. We instantiate 100 different system environments and calculate the sum rate of different groups of UEs in the system averaged over the 100 environments.

Fig. 4 gives the results of sum rates of FUEs under different FBS failure probabilities. Our proposed scheme shows up to $30 \%$ performance improvement compared to CSolu 1 and CSolu2. CSolu2 compensates the rate loss by handing all FUEs suffering from failure to MBS and PBS. CSolu1 allows FUEs suffering from failure to be handed over to other FBSs as well as MBS and PBS. CSolu2 outperforms CSolu1 in Fig. 4, which suggests that using available FBS resources to compensate rate loss without applying CoMP-JP, 


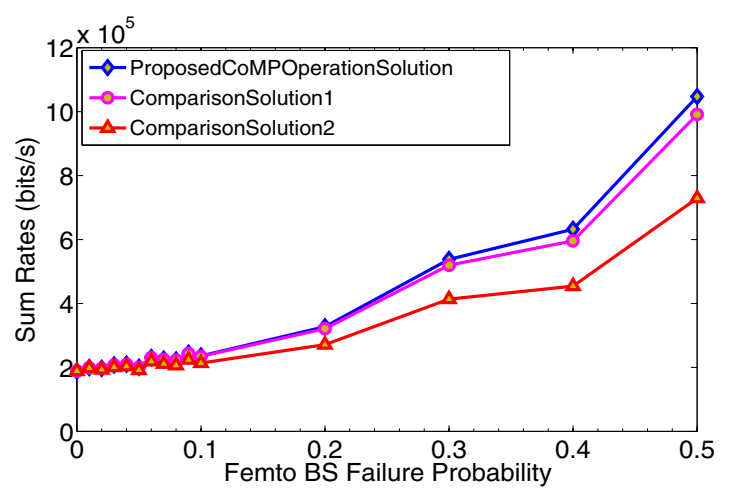

Fig. 6: Sum rates of non-FUEs within the residential apt units under different FBS failure probabilities.

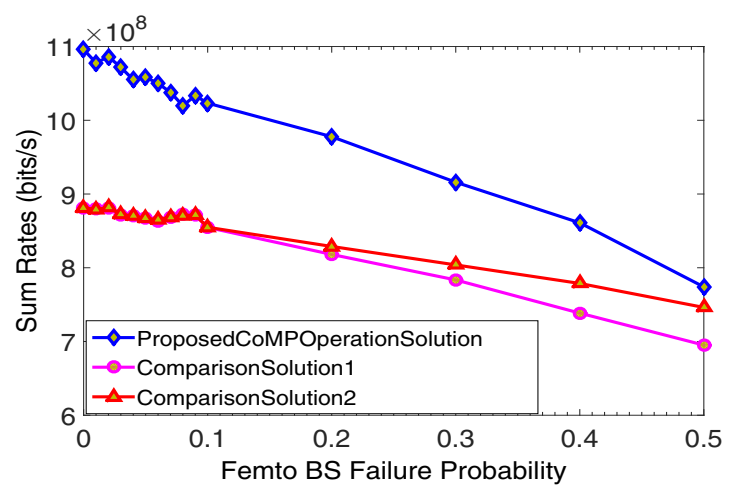

Fig. 7: System throughputs of all UEs under different FBS failure probabilities.

will cause more rate loss on FUEs sum rate than using other BS resources.

Table I lists the percentage of FUEs sum rate loss under different FBS failure probabilities with our proposed CoMPJP operation scheme. The results show that when FBS failure probability is up to $50 \%$, the FUEs sum rate has only $33.4 \%$ rate loss. Our proposed scheme can prevent rate loss from having the same speed of radio resource loss, mostly by using available FBS resources within the system.

Fig. 5 shows the results of sum rate of non-FUEs outside residential area. The failure of FBSs will decrease the interference suffered by these non-FUEs, hence resulting in rate increment. However, due to the failure of FBSs, more FUEs are handed over to MBS and PBS, resulting in resource blocks decrement to non-FUEs, hence resulting in rate loss. The decreasing trend of those rates along failure probabilities axis suggests that the non-FUEs rate loss due to resource blocks decrement per UE is more severe than the rate increment offered by decreased interference suffered from FBSs.

Fig. 6 shows that the sum UE rate of non-FUE subscribers within residential area will increase with the increment of FBS failure probability. Due to FBS failure, the suffered interference by non-FBSs within the residential apt units will decrease greatly. CSolu2 gives lower sum rate than our proposed solution and CSolu1, because when FBS fails, CSolu2 will hand over all FUEs suffering from failure to MBS and PBS, and results in more resource blocks reduction of non-
FBSs within residential area.

Fig 7 shows the total system throughputs with different FBS failure probabilities. The sum rates of all UEs in the system have the same trends as the FUEs sum rates shown in Fig. 4. Comparing the sum rates in Fig. 4, Fig. 5 and Fig. 6, we see that the sum rate of FUEs is much higher than both the sum rate of non-FUEs outside residential apt units, and non-FUEs within the residential apt units. This comparison shows that FBSs can provide higher rates to UEs than MBSs and PBSs. This justifies the increasing popularity of deployment of small cells in reality.

\section{CONCLUSION}

In this paper, we proposed a CoMP-JP operation scheme for FBSs to perform during both normal operation mode and cell outage compensation mode. we showed that our proposed CoMP-JP operation scheme provides a better performance comparing with other proposed solutions in the literature. Moreover, the proposed scheme proved the failure compensation capability by preventing the system sum rate loss from having the same speed of radio resource loss, and this is done without using additional radio resources and will not impair the performance of other UEs.

\section{REFERENCES}

[1] 3GPP, "TR 36.913: Requirements for further advancements for evolved universal terrestrial radio access (E-UTRA)," Available:www.3gpp.org, Tech. Rep., Mar. 2009.

[2] S. Hämäläinen, H. Sanneck, and C. Sartori, LTE self-organising networks (SON): Network management automation for operational efficiency. John Wiley \& Sons, Jan. 2012.

[3] M. Amirijoo, L. Jorguseski, R. Litjens, and L.-C. Schmelz, "Cell outage compensation in LTE networks: algorithms and performance assessment," in Proc. of the 73rd IEEE Vehicular Technology Conference (VTC Spring 2011), Yokohama, Japan, May 2011.

[4] F.-q. Li, X.-s. Qiu, L.-m. Meng, H. Zhang, and W. Gu, "Achieving cell outage compensation in radio access network with automatic network management," in Proc. of the IEEE Global Communications Conference Workshops (GC Wkshps 2011), Huston, TX, USA, Dec. 2011.

[5] M. Putzke and C. Wietfeld, "Self-organizing ad hoc femtocells for cell outage compensation using random frequency hopping," in Proc. of the 23rd IEEE International Symposium on Personal Indoor and Mobile Radio Communications (PIMRC 2012), Sydney, Australia, Sep. 2012.

[6] A. Apostolidis, D. Siouras, and N. Alonistioti, "An outage compensation algorithm for wlans," in Proc. of the International Conference on Smart Communications in Network Technologies (SaCoNeT), Paris, France, Jun. 2013.

[7] L. Kayili and E. Sousa, "Cell outage compensation for irregular cellular networks," in Proc. of the IEEE Wireless Communications and Networking Conference (WCNC 2014), Istanbul, Turkey, Apr. 2014.

[8] M. Sawahashi, Y. Kishiyama, A. Morimoto, D. Nishikawa, and M. Tanno, "Coordinated multipoint transmission/reception techniques for LTE-advanced [coordinated and distributed MIMO]," IEEE Wireless Communications, vol. 17, no. 3, pp. 26-34, Jun. 2010.

[9] 3GPP, "TR 36.819: Coordinated multi-point operation for LTE physical layer aspects (release 11)," Available:www.3gpp.org, Tech. Rep., Oct. 2011.

[10] 3GPP, “TR 36.814: Evolved universal terrestrial radio access, further advancements for E-UTRA physical layer aspects (release 9)," Available:www.3gpp.org, Tech. Rep., Mar. 2010.

[11] A. Wiesel, Y. C. Eldar, and S. Shamai, "Zero-forcing precoding and generalized inverses," IEEE Transactions on Signal Processing, vol. 56, no. 9, pp. 4409-4418, Aug. 2008.

[12] P. Baracca, F. Boccardi, and N. Benvenuto, "A dynamic clustering algorithm for downlink comp systems with multiple antenna ues," EURASIP Journal on Wireless Communications and Networking, vol. 2014, no. 1, pp. 1-14, Aug. 2014.

[13] C. Research, "CVX: A matlab-based convex modeling framework," Available:http://cvxr.com, last online access: Mar. 2015. 